\title{
Bonded molar tubes associated with higher failure rate than molar bands
}

\section{Abstracted from}

\section{Millett DT, Mandall NA, Mattick RC, Hickman J, Glenny AM.}

Adhesives for bonded molar tubes during fixed brace treatment. Cochrane Database Syst Rev. 2011 Jun 15: CD008236.

Address for correspondence: Luisa Fernandez Mauleffinch, Review Group Co-ordinator, Cochrane Oral Health Group, MANDEC, School of Dentistry, University of Manchester, Higher Cambridge Street, Manchester, M15 6FH, UK. E-mail: luisa.fernandez@manchester.ac.uk

\section{Question: How effective are adhesives used to attach bonded molar tubes or bands during fixed appliance treatment?}

Data sources The Cochrane Oral Health Group Trials Register, the Cochrane Central Register of Controlled Trials (CENTRAL), Medline, Embase, and Conference proceedings and abstracts from the British Orthodontic Conference, European Orthodontic Conference and the International Association of Dental Research were searched.

Study selection Randomised controlled clinical trials (RCT) including those that use a split-mouth design were included. There were no restrictions regarding the language.

Data extraction and synthesis The selection of papers, decisions about eligibility and data extraction were carried out independently and in duplicate without blinding to the authors, adhesives used or results obtained. All disagreements were resolved by discussion. Comparisons were to be made firstly between any of the five main types of adhesive. If possible, comparisons were to be made within groups and, where appropriate, between chemical- and light-cured adhesives.

Meta-analysis was to be undertaken only on studies of similar comparisons reporting the same outcome measures.

Results Two trials $(n=190)$, at low risk of bias, were included in the review and both presented data on first time failure at the tooth level. Pooling of the data showed a statistically significant difference in favour of molar bands, with a hazard ratio of 2.92 (95\% confidence intervals (Cl) 1.80 to 4.72 ). No statistically significant heterogeneity was shown between the two studies. Data on first time failure at the patient level were also available and showed a statistically different difference in favour of molar bands (risk ratio $2.30 ; 95 \% \mathrm{Cl} 1.56$ to 3.41 ) (risk of event for molar tubes $=57 \%$; risk of event for molar bands $25 \%$ ). One trial presented data on decalcification, again showing a statistically significant difference in favour of molar bands. No other adverse events were identified.

Conclusions From the two well-designed and low risk of bias trials included in this review it was shown that the failure of molar tubes bonded with either a chemically-cured or light-cured adhesive was considerably higher than that of molar bands cemented with glass ionomer cement. One trial indicated that there was less decalcification with molar bands cemented with glass ionomer cement than with bonded molar tubes cemented with a light-cured adhesive. However, given there are limited data for this outcome, further evidence is required to draw more robust conclusions.

\section{Commentary}

This systematic review presents data of high quality but low quantity. The proposed questions are of significant clinical importance and therefore of great interest for the readers. Generally accepted guidelines to conduct systematic reviews were followed and the available data represent a search up to the end of 2010. In essence, the available evidence clearly shows that molar bands in comparison to bonded tubes do offer a stronger resistance to bonding failures. The odds are approximately 3:1 (probability 66\%) that the cemented band will remain bonded throughout treatment compared to bonded tubes.

One of the factors that could have been considered in the analysis is where in the dental arch did the failure did happened. Clinical experience will tell us that lower arch molar tube failures are more common, but this was not analysed. An extra analysis in this regard would have been handy. Another factor of clinical interest would be the time of failure, ie is it more or less common after the first few weeks. Patients are usually told that bonded brackets are more likely to be lost during the first few days. Other factors like decalcification around attachments were considered and showed a clear advantage for the cemented molar bands, but because it comes from only one trial with a relatively small sample this needs to be interpreted with caution. Individual specific operator's experience and comfort with the utilised material are considered important.

Finally, due to the limited sample sizes it is not possible to determine if differences in failures are significantly related to specific cement types. The included studies both include glass ionomer cements and therefore any extrapolation to other type of resin based cements cannot be made. The authors notice that some factors like micro-etching and improved band/tube fitting to tooth surfaces may be important factors but the available data cannot permit a conclusion in this regard.

In summary, based on the included studies, cemented bands appear to provide distinct advantages both in retention and reduced decalcification in the surroundings. More specific analysis was not possible due to available sample sizes.

Carlos Flores-Mir Department of Dentistry, University of Alberta, Edmonton, Canada. Evidence-Based Dentistry (2011) 12, 84. doi:10.1038/sj.ebd.6400813 